ఠ

Open Access Full Text Article

REVIEW

\title{
Mindfulness training applied to addiction therapy: insights into the neural mechanisms of positive behavioral change
}

This article was published in the following Dove Press journal:

Neuroscience and Neuroeconomics

27 July 2016

Number of times this article has been viewed

\author{
Eric L Garland 1,2 \\ Matthew O Howard ${ }^{3}$ \\ Sarah E Priddy' \\ Patrick A McConnell ${ }^{4}$ \\ Michael R Riquino' \\ Brett Froeliger ${ }^{4}$ \\ 'College of Social Work, ${ }^{2}$ Hunstsman \\ Cancer Institute, University of Utah, \\ Salt Lake City, UT, USA; ${ }^{3}$ School of \\ Social Work, University of North \\ Carolina at Chapel Hill, Chapel \\ Hill, NC, USA; ${ }^{4}$ Department of \\ Neuroscience, Medical University of \\ South Carolina, Charleston, SC, USA
}

Correspondence: Eric L Garland College of Social Work, Huntsman Cancer Institute, 395 South, I500 East, University of Utah, Salt Lake City, UT 84II 2, USA

Email eric.garland@socwk.utah.edu

\begin{abstract}
Dual-process models from neuroscience suggest that addiction is driven by dysregulated interactions between bottom-up neural processes underpinning reward learning and top-down neural functions subserving executive function. Over time, drug use causes atrophy in prefrontally mediated cognitive control networks and hijacks striatal circuits devoted to processing natural rewards in service of compulsive seeking of drug-related reward. In essence, mindfulness-based interventions (MBIs) can be conceptualized as mental training programs for exercising, strengthening, and remediating these functional brain networks. This review describes how MBIs may remediate addiction by regulating frontostriatal circuits, thereby restoring an adaptive balance between these top-down and bottom-up processes. Empirical evidence is presented suggesting that MBIs facilitate cognitive control over drug-related automaticity, attentional bias, and drug cue reactivity, while enhancing responsiveness to natural rewards. Findings from the literature are incorporated into an integrative account of the neural mechanisms of mindfulness-based therapies for effecting positive behavior change in the context of addiction recovery. Implications of our theoretical framework are presented with respect to how these insights can inform the addiction therapy process.
\end{abstract}

Keywords: mindfulness, frontostriatal, savoring, cue reactivity, hedonic dysregulation, reward, addiction

\section{Automaticity and reward in addiction}

Drug addiction is a chronic relapsing brain disorder that involves dysregulated neural function in circuitry governing reward, learning, memory, and goal-directed behavior. The initial use of psychoactive substances is reinforced via dopaminergically mediated reward signaling in the ventral tegmental area. ${ }^{1}$ However, with repeated use, addictive substances impart motivational significance to drug-associated cues through an extended mesocorticolimbic brain circuit. ${ }^{2}$ Because obtaining and consuming psychoactive substances are motivationally salient goals in addiction, automatic drug-use action schemas $^{3}$ stored in memory guide implicit cognitive processing of stimuli associated with previous drug-use episodes. As such, drug-related cues come to evoke powerful conditioned motivational responses that may be unconscious and fully dissociable from the pleasure and reward previously elicited during the initial phases of drug use. ${ }^{4}$

Mechanistic research supports a dual-process model ${ }^{5}$ in which addiction is proposed to produce dysregulated interactions between bottom-up neural circuitry (eg, ventral striatum [VS]) that codes for the predictive value of a rewarding stimulus (eg, cigarette, food, sex $)^{6}$ and frontal-executive circuitry (eg, anterior cingulate cortex [ACC], lateral prefrontal cortex [lateral PFC]) that governs top-down cognitive control 
processes. ${ }^{7}$ Individuals with substance use disorders exhibit weaker functional connectivity between the aforementioned frontostriatal regions (eg, ACC $\rightarrow \mathrm{VS}),{ }^{8-10}$ the magnitude of which is associated with reduced self-reported positive affect, higher craving, and substance use following a quit attempt. ${ }^{11}$ Indeed, dysconnectivity between frontostriatal regions may account for the impairments in self-control and compulsive drug use that are hallmarks of addiction. Moreover, long-term exposure to drugs of abuse significantly attenuates neural response to naturally rewarding stimuli in the socioenvironment signaling homeostatic goal attainment (eg, eating, copulation, social affiliation), resulting in an overall hedonic deficit which may drive individuals to consume increasingly higher doses of the drug to maintain a dwindling sense of well-being. ${ }^{12}$

In summary, compulsive - or automatic - drug use is mediated by neuroplasticity in frontostriatal circuitry. ${ }^{13}$ The degree of integrity of functional communication in this circuit is shown to correspond to drug-related appetitive behaviors ${ }^{11}$ coupled with lack of responsivity to natural reward. ${ }^{14}$ Therefore, behavioral interventions that aim to interrupt automatized drug-use action schemas and restructure motivation and reward learning around non-drug-related natural rewards may be beneficial in helping individuals with substance use disorders maintain abstinence. By virtue of their therapeutic potential to target these two interrelated domains, mindfulness-based interventions (MBIs) may be efficacious for the treatment of addiction..$^{5,15}$

\section{Mindfulness as a state, trait, and practice}

Though mindfulness originated in contemplative practices in Asia over 2,500 years ago, this ancient concept is currently being examined by clinical researchers and neuroscientists alike for its promise in treating diverse psychological and physical conditions. ${ }^{16-19}$ Modern psychological research operationalizes the construct of mindfulness as a state, trait, and practice. Mindfulness may be conceptualized as a transient state of consciousness characterized by metacognitive awareness of present-moment cognition, emotion, sensation, and perception, without judgment or reactivity. ${ }^{16,18}$ Neuroimaging research suggests that generating the state of mindfulness may activate the dorsal ACC and dorsolateral PFC, ${ }^{20}$ which could facilitate metacognitive monitoring, attentional control, and regulation of affective reactivity. In doing so, the acute state of mindfulness may attenuate activation in brain areas that subserve negative affect (amygdala) and self-referential processing during emotional experience (eg, medial prefrontal cortex), while promoting interoceptive awareness in the face of affective challenges by increasing activation in the insula. ${ }^{21,22}$

The trait of mindfulness refers to a more enduring disposition or tendency to exhibit and express mindful attitudes and behaviors in everyday life, including nonreactivity to and acceptance of one's own momentary thoughts, feelings, and perceptions ${ }^{23,24}$ - individuals with no mindfulness meditation experience naturally vary in the extent to which they express this trait, the magnitude of which is negatively correlated with addiction vulnerability. ${ }^{24-26}$ Though traits are typically held to be relatively fixed attributes, there is evidence to suggest that the practice of mindfulness meditation can increase trait mindfulness, which in turn partially accounts for the therapeutic benefits of mindfulness practice. ${ }^{27}$ It has been hypothesized that the apparent malleability of trait mindfulness might stem from neuroplasticity induced by recurrent mindfulness practice. ${ }^{28,29} \mathrm{~A}$ body of research supports the notion that recurrent activation of the state of mindfulness might engender increased trait mindfulness via neuroplastic mechanisms. ${ }^{30}$ For instance, cross-sectional studies have demonstrated significant differences in gray matter volume between meditation practitioners and meditation-naïve controls, particularly in regions of PFC that instantiate cognitive control (eg, inferior frontal gyri) and higher order associative processing (eg, hippocampus). ${ }^{31-35}$ Moreover, longitudinal research has shown that participants in an 8-week MBI evidenced increased gray matter density in posterior cingulate cortex, temporoparietal junction, and cerebellum, compared to controls, ${ }^{29}$ and reduced amygdala volume that correlated with the degree of stress reduction achieved from mindfulness practice. ${ }^{36}$

The practice of mindfulness meditation involves the process of focusing attention on an object (eg, the sensation of breathing or walking, visual stimuli, or physical sensations in the body) while acknowledging and, in turn, letting go of distracting thoughts and emotions. ${ }^{37}$ These focused attention practices are complemented by open monitoring forms of mindfulness meditation during which practitioners metacognitively observe their psychological experience, that is, focusing on the quality of awareness itself as opposed to any particular content of awareness. ${ }^{18}$ These practices of focused attention and open monitoring activate different cognitive functions, including attentional vigilance, attentional reorienting, executive monitoring of working memory, response inhibition, and emotion regulation. ${ }^{38}$ Often, they are combined into a single meditation that begins with focused attention and evolves into open monitoring. Contemporary 
MBIs like mindfulness-based stress reduction, ${ }^{39}$ which provide training in a range of mindfulness practices (eg, mindful breathing, body scan techniques), have been shown to produce therapeutic effects for a variety of clinical conditions, including depression, anxiety, and chronic pain. ${ }^{40,41}$

Germane to the present review, MBIs may also target the mechanisms underlying addiction, including attentional automaticity toward drug-related cues and attenuated natural reward processing, by teaching mindfulness practices designed to induce the state of mindfulness, and thereby enhance the trait of mindfulness in everyday life. Among MBIs, Mindfulness-Based Relapse Prevention ${ }^{42,43}$ and Mindfulness-Oriented Recovery Enhancement ${ }^{44,45}$ have demonstrated efficacy in treating addiction. Results from controlled trials of these and other MBIs have demonstrated significant therapeutic effects among individuals with alcohol, ${ }^{46}$ drug, ${ }^{47,48}$ nicotine, ${ }^{49}$ and prescription opioid ${ }^{45,50}$ use disorders. Through mindfulness training, MBIs foster the development of state and trait mindfulness, and thereby may alter neural circuits subserving the habit and reward functions that drive addictive behaviors.

\section{Effects of mindfulness on automaticity and attentional bias}

As previously discussed, the initiation of drug use is marked by euphoric experiences in response to drug administration. This euphoria is coupled with strong positive reinforcement to continue seeking and consuming drugs. Over time, chronic drug use becomes an overlearned, automatic process, undergirded by neuroplastic changes in striatal circuitry subserving automatized habit behavior. ${ }^{51,52}$

In contrast to such addictive automaticity, mindfulness involves conscious awareness of automatized cognitive, emotive, and behavioral responses. MBIs focus on helping individuals develop awareness of automatic behavior in daily living by bringing full consciousness to the sensorimotor experiences of repetitive tasks. As such, mindfulness may promote regulation of processes linked to automatic addictive behavior. In support of this possibility, an early study of the neural mechanisms of meditation found meditation-induced increases in dopamine release in the $\mathrm{VS},{ }^{53}$ which suggests that MBIs may target striatal dopamine transmission, and thereby modulate neurobiologic processes underpinning automaticity. Furthermore, the pro-regulatory effects of mindfulness on automaticity have been supported by behavioral studies, which indicate that mindfulness training decreases habitual responses on Stroop tasks ${ }^{54}$ and reduces rigid adherence to scripted cognitive responses. ${ }^{55}$ Specific to automaticity in addiction, MBIs may interrupt drug-use action schemas. When such schemas are activated by substance-related cues or negative emotion, individuals who have developed trait mindfulness as a result of mindfulness practice may become aware of the resulting automatic urge to consume drugs. Then, by evoking state mindfulness through focused attention or open monitoring forms of mindfulness meditation, the individual may be able to interrupt the automatized response and instead utilize an intentional coping response. This notion was supported by $\mathrm{McHugh}$ et al, ${ }^{56}$ who found that a brief mindfulness induction increased awareness of implicit cues and reduced reactivity to previously learned implicit associations. This process may be facilitated by recruiting top-down executive control via a frontoparietal metacognitive attention network, which may lead to increased self-regulation and prevention of substance use.

A second way in which mindfulness might modulate addictive automaticity is by increasing control over attentional bias toward drug-related cues. Substance-dependent individuals exhibit attentional biases to drug-related cues due to the emotional and cognitive salience of such stimuli. ${ }^{57}$ Through incentive sensitization via the mesocorticolimbic dopamine system, chronic substance users are biased to attend to drug cues more readily than neutral cues - manifested in nonvolitional attentional capture by drug cues and/ or the inability to disengage and shift attention from those cues. Such addiction attentional biases can significantly predict relapse following treatment, even after controlling for factors like craving and addiction severity. ${ }^{46,58,59} \mathrm{MBIs}$ may target addiction attentional bias by promoting attentional reorienting capacity. ${ }^{60,61}$ Mindfulness practice may strengthen attentional reorienting through mindful breathing and body scan practices which involve noticing cognitive distraction and then returning the focus of attention back to presentmoment somatic sensations (like breathing). This intentional, recurrent engagement and subsequent disengagement of attention may strengthen frontoparietal network function to improve regulation of automatic attentional processes. Using mindfulness techniques to repeatedly reorient attention from drug-related cues to neutral or healthful stimuli may extinguish conditioned associations between drug-related cues and drug-use action schemas which drive compulsive drug use. In this regard, ${ }^{62}$ it was observed that long-term meditators were better able to attenuate conditioned reward expectations associated with learned cues by decreasing activation in the striatum.

Taken together, prior studies suggest that mindfulness training facilitates frontal-executive regulatory processes, 
including 1) deautomatization of habitual cognitive and behavioral processes and 2) augmentation of attentional reorienting capacity. These frontal-executive benefits of mindfulness training may explain the positive effects of MBIs on attentional bias among individuals with alcohol use disorders ${ }^{46}$ and extended histories of prescription opioid use, ${ }^{63,64}$ suggesting that mindfulness training might be useful in disrupting and restructuring automatic reactivity to drug cues. We discuss this subject in the following section.

\section{Effects of mindfulness on drug cue reactivity and natural reward sensitivity}

In addition to the cognitive effects outlined earlier, mindfulness practice has a long-standing reputation for increasing emotional well-being; ${ }^{40}$ yet, the neuroscientific literature supporting this popular notion is still in a nascent phase of development. ${ }^{19}$ Buddhist monks - putative experts in mindfulness-based meditative practices - and other advanced practitioners (individuals with $>10,000$ hours of meditation practice) have increasingly become a population of interest for neuroimaging studies investigating the neural bases of mindfulness-based changes in the regulation of affective experience. For instance, a neuroimaging case study examined an advanced practitioner using electroencephalogram and functional magnetic resonance imaging while the subject performed Jhana meditation (a form of ecstatic meditation aimed at increasing positive affect). These data showed that the emotional bliss and altered states of consciousness produced by this meditative technique were associated with robust activations within frontostriatal reward circuitry. ${ }^{65}$ Such augmentation of neural reward circuit function might explain meta-analytic findings of increased positive emotions among practitioners of affect-focused meditation techniques ${ }^{66}$ and mindfulness practices, ${ }^{67-69}$ implicating a role for MBIs in restructuring reward processes dysregulated by addiction.

Certainly, not all forms of mindfulness-based practice are employed to generate positive affect as a primary shortterm goal. Rather, mindfulness is often seen more secularly as a means of calming the mind, decentering from habitual thoughts and reactions, and making "mental space" through which novel insights or mindful reappraisals might arise. Nevertheless, this does not mean that a technique must be affect-focused for a restructuring of reward processing to occur over repeated practice. One recently proposed theoretical model, the mindfulness to meaning theory (MMT), ${ }^{70}$ explores potential mindfulness-based mechanisms that may contribute to the restructuring of reward processing and affective responses in practitioners. To this end, MMT presents a generalized dual-component hypothesis of mindfulness-based attention-affect interaction, proposing that neural reward circuitry adaptations transpire through 1) disengagement from maladaptive internal or external stimuli (attentional shift) and 2) subsequent engagement with healthful or naturally rewarding stimuli to amplify their affective valence or salience (savoring). ${ }^{70}$

During an attentional shift, mindfulness practice is thought to redirect attention away from maladaptive cognitions such as rumination or craving. In doing so, redirection frees attentional resources for more flexible deployment and widens the field of attention to include the broader socioemotional context of the immediate experience, thus making space for adaptive positive reappraisals. ${ }^{70}$ By reallocating attentional resources that are freed during an attentional shift, the practitioner can engage in the amplification of positive affect resulting from the perception of newly salient natural rewards (ie, increased natural reward sensitivity $\rightarrow$ savoring). Through the process of savoring, the authors of MMT posit that mindfulness-based adaptations to neural reward circuitry (eg, frontostriatal, striatal-insular networks) that retune the system toward natural rewards may help to counter accumulated allostatic load resulting from lifetime stress and/or addiction. ${ }^{7,52}$

Such restructuring of reward responding may be instantiated by the dynamic reconfiguration of neural networks observed during meditation (ie, meditation-state functional connectivity), including increased coupling between the default mode network, dorsal attention network, and salience network - regions subserving attentional control and reward processing. ${ }^{71}$ Furthermore, accumulated meditative practice may also alter resting-state functional connectivity $^{72}$ that could underpin long-term increases in trait mindfulness. These reconfigurations in connectivity patterns may explicate findings from an affective Stroop task demonstrating that experienced meditators exhibit a decoupling of affective response from limbic reactivity to negative emotional stimuli. ${ }^{53}$ With respect to reconfigurations of neural circuit function during reward processing, mindfulness practitioners exhibited striatal blood-oxygen level dependent responses during an early-stage natural reward conditioning task similar to control subjects, but the practitioners demonstrated decreased striatal activation in response to reward prediction error paralleled by increased blood-oxygen level dependent signal in posterior insula. ${ }^{62}$

In the context of addiction, there is evidence that mindfulness practice may reduce drug cue reactivity, while 
concomitantly increasing natural reward responsiveness. ${ }^{73}$ A recent randomized controlled trial of Mindfulness-Oriented Recovery Enhancement (MORE) demonstrated that mindfulness training decoupled drug craving from addictive behaviors $^{46}$ and significantly reduced subjective and autonomic indices of drug cue reactivity. ${ }^{73}$ Concomitantly, MORE led to enhanced parasympathetic nervous system responses (a physiological marker of appetitive processes and positive affect) to stimuli representing natural rewards that mediated the effects of MORE on drug craving. ${ }^{73}$ Furthermore, event-related potential data from this study indicate that the MORE intervention enhanced electroencephalogram correlates of natural reward processing, which were associated with increased positive affective responses to natural reward cues and reduced drug craving. ${ }^{50}$

Preliminary neuroimaging evidence suggests that MORE may restructure reward processing by treating dysfunction in frontostriatal circuits integral to addiction. In a functional magnetic resonance imaging analysis of a pilot study of MORE as a treatment for nicotine addiction, ${ }^{49}$ smokers completed a cue-reactivity task in which cigarette images were viewed and a positive emotion regulation task in which participants were asked to either view a positive image or upregulate positive affective response to the image via savoring. On the cue-reactivity task, significant group $\times$ time effects were observed in the VS, such that participants in the MORE intervention exhibited significant decreases in striatal responses to cigarette cues over time, relative to those in the no-treatment control condition. In contrast, during the emotion regulation task, significant group $\times$ time effects were observed in the VS and rostral ACC for savor trials relative to view trials, such that compared to the no-treatment control group, participation in MORE was associated with increased response in the VS and rostral ACC over time. Finally, increases in striatal and ACC savoring responses significantly predicted increases in positive affect and decreases in the number of cigarettes smoked during the same time frame.

To summarize, the literature suggests that mindfulness practitioners appear to exhibit: 1) increased positive affect, 2) altered functional connectivity in neural cognitive control and reward circuitry at rest and during meditation, 3 ) decoupling of affective reactivity from subsequent mood states and behaviors, 4) reduced drug cue reactivity, and 5 ) improved natural reward processing. Therefore, the dualcomponent effects described earlier make the formal study of prospective applications of mindfulness practice highly relevant to developing novel and theoretically grounded therapies for addictive disorders, especially as pertaining to remediation of dysregulated top-down control over affective processes. $^{74}$

\section{An integrative model of the neural mechanisms of by which mindfulness promotes positive behavior change in addiction}

Given the aforementioned findings and hypotheses, we present the following integrative model of the neural mechanisms by which mindfulness might promote positive behavior change in addiction. To reiterate, over time, drug use causes atrophy in prefrontally mediated cognitive control networks and hijacks striatal circuits devoted to processing natural rewards in service of compulsive seeking of drug-related reward. In essence, MBIs can be conceptualized as mental training programs for exercising, strengthening, and remediating these functional brain networks. Such mental training, when sustained over time and with sufficient intensity, may ultimately result in the changes in brain structure found in meta-analyses of the neuroanatomical effects of meditation ${ }^{31}$ and discussed earlier.

Specifically, we propose that mindfulness training augments functional connectivity between prefrontal, cingulate, parietal, and thalamic nodes of an executive regulatory circuit that provides feedback to the striatum and medial temporal lobe concerning interoceptive representations of the reward value of various courses of action and their alignment with one's overall goal orientation. The functional output of this mindfulness-centered regulatory network (Figure 1) may allow individuals to gain awareness of automatized behavioral repertoires, restructure conditioned associations, and maintain an optimal hedonic tone to support healthy functioning.

A number of studies support various aspects of the integrative model. First, mindfulness training appears to increase connectivity within the insula, ${ }^{75}$ a key node of the salience network, suggesting that mindfulness may be able to alter interoceptive representations to optimize reward prediction error in service of adaptive behavior. ${ }^{76,77}$ Second, mindfulness training is associated with augmented connectivity to dorsal attentional control regions, ${ }^{71,78}$ allowing for increased top-down conscious regulation of automatized bottom-up responses. Finally, during meditation, strengthening of connectivity has been observed between dorsal attention network, salience network, and default mode network nodes, ${ }^{71}$ providing a potential avenue by which metacognition might self-regulate intrinsic associative mentation, ${ }^{79}$ and thereby restructure the higher order meaning and value of drug and natural rewards. Such 


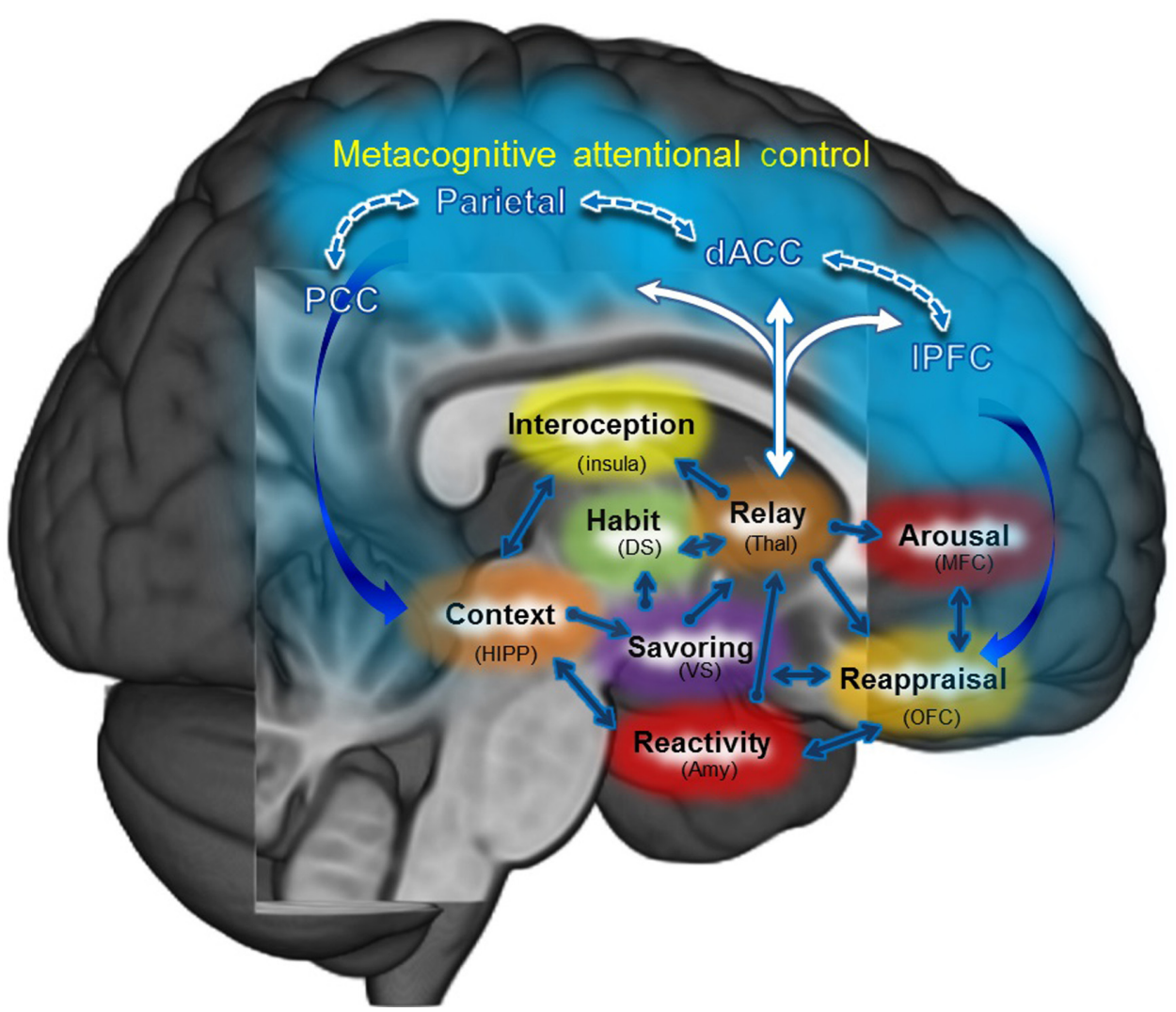

Figure I Mindfulness-centered regulatory network.

Notes: The central tenet of this model posits that mindfulness-based interventions may remediate dysregulation in neural circuits integral to addiction by way of strengthening functional connectivity: I) within a metacognitive attentional control network (dIPFC, dACC, parietal cortex); and 2) between the metacognitive attentional control network and brain regions subserving automatic habit behavior, memory consolidation, interoceptive awareness, and reward processing. Reproduced from Garland EL, Froeliger B, Howard MO. Mindfulness training targets neurocognitive mechanisms of addiction at the attention-appraisal-emotion interface. Front Psychiatry. 20I3;4:I73. ${ }^{5}$

Abbreviations: dIPFC, dorsolateral prefrontal cortex; dACC, dorsal anterior cingulate cortex; PCC, posterior cingulate cortex; DS, dorsal striatum; VS, ventral striatum; Thal, thalamus; HIPP, hippocampus; Amy, amygdala; OFC, orbitofrontal cortex; MFC, medial prefrontal cortex; IPFC, lateral prefrontal cortex.

enhanced functional connectivity may be underpinned by neuroplastic anatomical changes integral to efficient neural communication. In this regard, a randomized controlled trial demonstrated that 10 hours of meditation training can increase white matter efficiency in the corona radiata, a white matter tract connecting the ACC to other brain structures, ${ }^{80}$ which was apparently mediated by increased myelination and axonal changes. ${ }^{81}$ More controlled studies of the acute and long-term effects of mindfulness meditation on functional and structural brain connectivity are needed to support our integrative network model of mindfulness-centered regulation.

From a clinical perspective, we hypothesize that activation of this network may support addiction recovery by fostering positive behavior change in a number of respects. First, when cortico-limbic-striatal circuits subserving drug-use action schemas are activated by cues associated with past drug-use episodes, mindfulness may engender awareness of the automatic addictive habit, as it is activated via insula-mediated interoceptive awareness, allowing for effective and timely top-down prefrontal regulation of the bottom-up appetitive urge. When attention is captivated by addictive cues, formal mindfulness meditation practices (eg, mindful breathing) can be used to regulate frontoparietal attentional networks, facilitating disengagement from drug cues and subsequent engagement of attention to neutral somatic sensations (eg, respiration). During this process, the individual may metacognitively monitor cravings as they arise and reappraise them as ephemeral, insubstantial mental phenomena, instead of perceiving them as inexorable compulsions for action. When mindfulness of craving is sustained over time without drug use, craving sensations may abate, as extinction learning weakens associative linkages between conditioned addiction-related stimuli and the attendant conditioned appetitive response. Such decoupling of urge from action may stem from prefrontal downregulation of limbic-striatal activation.

Furthermore, once mindfulness has interrupted the ballistic action of drug-use action schema, it can be used to stabilize attention on mental simulations of potential future adverse consequences of drug use. When mind wandering 
occurs, mindfulness can be used to reorient attention back to the mental simulation of these consequences, elaborating on them and gradually building a wider network of associations, until through aversive counterconditioning, the urge to get high, which was once so compelling, is now experienced as repugnant. This process may rely, in part, upon medial prefrontal $\rightarrow$ hippocampal updating of context-dependent associations.

In addition to disrupting prior conditioned associations, mindfulness may be used to restore and build novel associations to non-drug-related rewards. Through mindful savoring practices, naturally rewarding experiences that had lost their luster during the allostatic process of addiction may again be reinvested with salience. In other words, the addict can relearn the pleasures of sober social affiliation, the sense of accomplishment from a job well done, or the profound peace and contentment that comes from basking in the beauty of nature. To facilitate this relearning of natural reward, mindfulness meditation can be used to orient and sustain attention on the pleasant sensory-perceptual features of healthful objects and events while using attention to amplify any positive emotions or affective meaning arising during the savoring experience. Such intense attentional focus can be implemented without clinging, thereby fostering a deep appreciation for positive experience, no matter how fleeting. This technique may increase orbital-frontal and striatal responses to cues signaling natural rewards, thereby motivating engagement in healthy behaviors that subserve goal attainment. Ultimately, mindfulness practice may enhance the capacity to savor natural rewards and regulate drug craving by developing the malleable trait of mindfulness via neuroplasticity induced by recurrent brain state switching from mindless, addictive automaticity to the mindful state.

Though the original Buddhist soteriological purpose of mindfulness was to foster insight into the impermanent and interdependent nature of mental experience, modern MBIs may benefit individuals with substance use disorders by fostering addiction recovery and effecting positive behavior change. Through repeated practice of regulating addictive responses and extracting pleasure from life without substance use, the process of mindful recovery may ultimately reestablish healthy dopaminergic tone and remediate frontostriatal network function that had become dysregulated during the process of addiction.

\section{Acknowledgments}

ELG was supported by grant R34DA037005 from the National Institutes of Health (NIH) in preparing this manuscript. The conclusions in this article are those of the authors and do not necessarily represent the official position of the NIH.

\section{Disclosure}

The authors report no conflicts of interest in this work.

\section{References}

1. Feltenstein MW, See RE. The neurocircuitry of addiction: an overview. Br J Pharmacol. 2008;154(2):261-274. doi:10.1038/bjp.2008.51.

2. Robinson TE, Berridge KC. The incentive sensitization theory of addiction: some current issues. Philos Trans $R$ Soc B Biol Sci. 2008;363(1507):3137-3146. doi:10.1098/rstb.2008.0093.

3. Tiffany ST. A cognitive model of drug urges and drug-use behavior: role of automatic and nonautomatic processes. Psychol Rev. 1990;97(2):147-168.

4. O'Brien CP, Childress AR, Ehrman R, Robbins SJ. Conditioning factors in drug abuse: can they explain compulsion? J Psychopharmacol (Oxf). 1998;12(1):15-22.

5. Garland EL, Froeliger B, Howard MO. Mindfulness training targets neurocognitive mechanisms of addiction at the attention-appraisalemotion interface. Front Psychiatry. 2013;4:173.

6. Knutson B, Gibbs SE. Linking nucleus accumbens dopamine and blood oxygenation. Pscyhopharmacology (Berl). 2007;191(3):813-822. doi:10.1007/s00213-006-0686-7.

7. Goldstein RZ, Volkow ND. Drug addiction and its underlying neurobiological basis: neuroimaging evidence for the involvement of the frontal cortex. Am J Psychiatry. 2002;159(10):1642-1652.

8. Hong LE, Gu H, Yang Y, et al. Association of nicotine addiction and nicotine's actions with separate cingulate cortex functional circuits. Arch Gen Psychiatry. 2009;66(4):431-441. doi:10.1001/archgenpsychiatry. 2009.2.

9. Ma N, Liu Y, Li N, et al. Addiction related alteration in resting-state brain connectivity. Neuroimage. 2010;49(1):738-744.

10. Motzkin JC, Baskin-Sommers A, Newman JP, Kiehl KA, Koenigs M. Neural correlates of substance abuse: reduced functional connectivity between areas underlying reward and cognitive control. Hum Brain Mapp. 2014;35(9):4282-4292. doi:10.1002/hbm.22474.

11. Froeliger B, McConnell PA, Stankeviciute N, McClure EA, Kalivas PW, Gray KM. The effects of N-acetylcysteine on frontostriatal resting-state functional connectivity, withdrawal symptoms and smoking abstinence: A double-blind, placebo-controlled fMRI pilot study. Drug Alcohol Depend. 2015;156:234-242. doi:10.1016/j.drugalcdep.2015.09.021.

12. Koob GF, LeMoal M. Drug addiction, dysregulation of reward, and allostasis. Neuropscyhopharmacology. 2001;24(2):97-129. doi:10.1016/S0893-133X(00)00195-0.

13. Kalivas PW. The glutamate homeostasis hypothesis of addiction. Nat Rev Neurosci. 2009;10(8):561-572. doi:10.1038/nrn2515.

14. Forbes EE, Rodriguez EE, Musselman S, Narendran R. Prefrontal response and frontostriatal functional connectivity to monetary reward in abstinent alcohol-dependent young adults. PLoS One. 2014;9(5):e94640. doi:10.1371/journal.pone.0094640.

15. McConnell PA, Froeliger B. Mindfulness, mechanisms and meaning: perspectives from the cognitive neuroscience of addiction. Psychol Inq. 2015;26(4):349-357. doi:10.1080/1047840X.2015.1076701.

16. Garland EL. The meaning of mindfulness: A second-order cybernetics of stress, metacognition, and coping. Complement Health Pract Rev. 2007;12:15-30.

17. Goyal M, Singh S, Sibinga EMS. Meditation programs for psychological stress and well-being. JAMA Intern Med. 2014;174(3):357-368. doi:10.1001/jamainternmed.2013.13018.

18. Lutz A, Slagter HA, Dunne JD, Davidson RJ. Attention regulation and monitoring in meditation. Trends Cogn Sci. 2008;12: 163-169.

19. Tang YY, Hölzel BK, Posner MI. The neuroscience of mindfulness meditation. Nat Rev Neurosci. 2015;16:213-225. doi:10.1038/ nrn3916. 
20. Chiesa A, Serretti A. A systematic review of neurobiological and clinical features of mindfulness meditations. Psychol Med. 2010;40: 1239-1252.

21. Farb NA, Anderson AK, Mayberg H, Bean J, McKeon D, Segal ZV. Minding one's emotions: mindfulness training alters the neural expression of sadness. Emotion. 2010;10:25-33.

22. Taylor VA, Grant J, Daneault V, et al. Impact of mindfulness on the neural responses to emotional pictures in experienced and beginner meditators. NeuroImage. 2011;57(4):1524-1533. doi:10.1016/j. neuroimage.2011.06.001.

23. Baer RA, Smith GT, Hopkins J, Krietemeyer J, Toney L. Using selfreport assessment methods to explore facets of mindfulness. Assessment. 2006;13(1):27-45.

24. Garland EL, Boettiger CA, Howard MO. Targeting cognitive-affective risk mechanisms in stress-precipitated alcohol dependence: an integrated, biopsychosocial model of automaticity, allostasis, and addiction. Med Hypotheses. 2011;76(5):745-754.

25. Garland EL, Boettiger CA, Gaylord S, Chanon VW, Howard MO. Mindfulness is inversely associated with alcohol attentional bias among recovering alcohol-dependent adults. Cogn Ther Res. 2012:36(5): $441-450$.

26. Garland EL, Roberts-Lewis A, Kelley K, Tronnier C, Hanley A. Cognitive and affective mechanisms linking trait mindfulness to craving among individuals in addiction recovery. Subst Use Misuse. 2014;49(5):525-535. doi:10.3109/10826084.2014.850309.

27. Carmody J, Baer RA. Relationships between mindfulness practice and levels of mindfulness, medical and psychological symptoms and wellbeing in a mindfulness-based stress reduction program. J Behav Med. 2008;31:23-33.

28. Garland EL, Fredrickson BL, Kring AM, Johnson DP, Meyer PS, Penn DL. Upward spirals of positive emotions counter downward spirals of negativity: Insights from the broaden-and-build theory and affective neuroscience on the treatment of emotion dysfunctions and deficits in psychopathology. Clin Psychol Rev. 2010;30:849-864.

29. Holzel BK, Carmody J, Vangel M, et al. Mindfulness practice leads to increases in regional brain gray matter density. Psychiatry Res. 2011;191:36-43.

30. Fox KC, Nijeboer S, Dixon ML, et al. Is meditation associated with altered brain structure? A systematic review and meta-analysis of morphometric neuroimaging in meditation practitioners. Neurosci Biobehav Rev. 2014;43:48-73.

31. Froeliger B, Garland EL, McClernon FJ. Yoga meditation practitioners exhibit greater gray matter volume and fewer reported cognitive failures: results of a preliminary voxel-based morphometric analysis. Evid Based Complement Alternat Med. 2012;2012. Available from: http:// www.hindawi.com.proxy.lib.fsu.edu/journals/ecam/2012/821307/abs/. Accessed May 20, 2013.

32. Holzel BK, Ott U, Gard T, et al. Investigation of mindfulness meditation practitioners with voxel-based morphometry. Soc Cogn Affect Neurosci. 2008;3:55-61.

33. Lazar SW, Kerr CE, Wasserman RH, et al. Meditation experience is associated with increased cortical thickness. Neuroreport. 2005;16:1893-1897.

34. Luders E, Toga AW, Lepore N, Gaser C. The underlying anatomical correlates of long-term meditation: larger hippocampal and frontal volumes of gray matter. Neuroimage. 2009;45(3):672-678.

35. Kang DH, Jo HJ, Jung WH, et al. The effect of meditation on brain structure: cortical thickness mapping and diffusion tensor imaging. Soc Cogn Affect Neurosci. Epub 2012 May 7. doi:10.1093/scan/nss056.

36. Hölzel BK, Carmody J, Evans KC, et al. Stress reduction correlates with structural changes in the amygdala. Soc Cogn Affect Neurosci. 2010;5(1):11-17.

37. Lutz A, Dunne JD, Davidson RJ. Meditation and the neuroscience of consciousness: an introduction. In: Zelazo PD, Moscovitch M, Thompson E, editors. The Cambridge Handbook of Consciousness. New York: Cambridge University Press; 2007:497-549.
38. Vago DR, Silbersweig DA. Self-awareness, self-regulation, and self-transcendence (S-ART): A framework for understanding the neurobiological mechanisms of mindfulness. Front Hum Neurosci. 2012;6(296):1-30.

39. Kabat-Zinn J. Wherever You Go, There You Are: Mindfulness Meditation in Everyday Life. New York, NY: Hyperion Books; 1994.

40. Goyal M, Singh S, Sibinga EM, et al. Meditation programs for psychological stress and well-being: a systematic review and meta-analysis. JAMA Intern Med. 2014;174(3):357-368.

41. Chiesa A, Serretti A. Mindfulness-based interventions for chronic pain: a systematic review of the evidence. JAltern Complement Med. 2011;17(1):83-93. doi:10.1089/acm.2009.0546.

42. Bowen S, Chawla N, Collins SE, et al. Mindfulness-based relapse prevention for substance use disorders: A pilot efficacy trial. Subst Abuse. 2009;30:295-305.

43. Bowen S, Chawla N, Marlatt GA. Mindfulness-Based Relapse Prevention for Addictive Behaviors. New York: Guilford Press; 2010.

44. Garland EL. Mindfulness-Oriented Recovery Enhancement: Reclaiming a Meaningful Life from Addiction, Stress, and Pain. Washington, DC: NASW Press; 2013.

45. Garland EL, Manusov EG, Froeliger B, Kelly A, Williams JM, Howard MO. Mindfulness-Oriented Recovery Enhancement for chronic pain and prescription opioid misuse: results from an early-stage randomized controlled trial. J Consult Clin Psychol. 2014;82(3): 448-459.

46. Garland EL, Gaylord SA, Boettiger CA, Howard MO. Mindfulness training modifies cognitive, affective, and physiological mechanisms implicated in alcohol dependence: results of a randomized controlled pilot trial. J Psychoactive Drugs. 2010;42(2):177-192.

47. Garland EL, Roberts-Lewis A, Tronnier C, Graves R, Kelley K. Mindfulness-Oriented Recovery Enhancement versus CBT for co-occurring substance dependence, traumatic stress, and psychiatric disorders: proximal outcomes from a pragmatic randomized trial. Behav Res Ther. 2016;77:7-16. doi:10.1016/j.brat.2015.11.012.

48. Bowen S, Witkiewitz K, Clifasefi SL, et al. Relative efficacy of mindfulness-based relapse prevention, standard relapse prevention, and treatment as usual for substance use disorders: a randomized clinical trial. JAMA Psychiatry. 2014;71(5):547-556. doi:10.1001/ jamapsychiatry.2013.4546.

49. Eichberg C, Mathew A, Baddeley J, et al. Mindfulness-Oriented Recovery Enhancement (MORE): a novel smoking-cessation treatment focused on cognitive reappraisal and the savoring of naturalistic rewards. Poster presented at: Frontiers in Neuroscience; April 6, 2015; Charleston, SC.

50. Garland EL, Froeliger B, Howard MO. Neurophysiological evidence for remediation of reward processing deficits in chronic pain and opioid misuse following treatment with Mindfulness-Oriented Recovery Enhancement: exploratory ERP findings from a pilot RCT. J Behav Med. 2014;38(2):327-336. doi:10.1007/s10865-0149607-0.

51. Ito R, Dalley JW, Robbins TW, Everitt BJ. Dopamine release in the dorsal striatum during cocaine-seeking behavior under the control of a drug-associated cue. J Neurosci. 2002;22:6247-6253.

52. Volkow ND, Wang GT, Telang F, et al. Dopamine increases in striatum do not elicit craving in cocaine abusers unless they are coupled with cocaine cues. Neuroimage. 2008;39:1266-1273. doi:10.1016/j. neuroimage.2007.09.059.

53. Kjaer TW, Bertelsen C, Piccini P, Brooks D, Alving J, Lou HC. Increased dopamine tone during meditation-induced change of consciousness. Brain Res Cogn Brain Res. 2002;13:255-259. doi:10.1016/S09266410(01)00106-9.

54. Wenk-Sormaz H. Meditation can reduce habitual responding. Altern Ther Health Med. 2005;11:42-58.

55. Greenberg J, Reiner K, Meiran N. "Mind the trap": mindfulness practice reduces cognitive rigidity. PLoS One. 2012;7:e36206. doi:10.1371/ journal.pone. 0036206 . 
56. McHugh L, Procter J, Herzog M, Schock AK, Reed P. The effect of mindfulness on extinction and behavioral resurgence. Learn Behav. 2012;40(4):405-415.

57. Field M, Cox WM. Attentional bias in addictive behaviors: a review of its development, causes, and consequences. Drug Alcohol Depend. 2008;97:1-20.

58. Garland EL, Franken IH, Howard MO. Cue-elicited heart rate variability and attentional bias predict alcohol relapse following treatment. Psychopharmacology (Berl). 2012;222(1):17-26.

59. Schoenmakers TM, de Bruin M, Lux IF, Goertz AG, Van Kerkhof DH, Wiers RW. Clinical effectiveness of attentional bias modification training in abstinent alcoholic patients. Drug Alcohol Depend. 2010;109:30-36.

60. Jha A, Krompinger J, Baime M. Mindfulness training modifies subsystems of attention. Cogn Affect Behav Neurosci. 2007;7:109-119.

61. van den Hurk PAM, Giommi F, Gielen SC, Speckens AEM, Barendregt HP. Greater efficiency in attentional processing related to mindfulness meditation. Q J Exp Psychol. 2010;63(6):1168-1180.

62. Kirk U, Montague PR. Mindfulness meditation modulates reward prediction errors in a passive conditioning task. Front Psychol. 2015;6:90.

63. Garland EL, Howard MO. Mindfulness-Oriented Recovery Enhancement reduces pain attentional bias in chronic pain patients. Psychother Psychosom. 2013;82:311-318. doi:10.1159/000348868.

64. Garland EL. Mindfulness-Oriented Recovery Enhancement modulates neurocognitive mechanisms and reward system function in addiction, stress, and pain. Invited presentation at: Advances in Meditation Research; September 25, 2015; Sloan Kettering Memorial Hospital, New York, NY.

65. Hagerty MR, Isaacs J, Brasington L, Shupe L, Fetz EE, Cramer SC. Case study of ecstatic meditation: fMRI and EEG evidence of self-stimulating a reward system. Neural Plast. Epub 2013 May 2. doi:10.1155/2013/653572.

66. Zeng X, Chiu CP, Wang R, Oei TP, Leung FY. The effect of lovingkindness meditation on positive emotions: a meta-analytic review. Front Psychol. 2015;6:1693.

67. Davis MC, Zautra AJ. An online mindfulness intervention targeting socioemotional regulation in fibromyalgia: results of a randomized controlled trial. Ann Behav Med. 2013;46(3):273-284.

68. Geschwind N, Peeters F, Drukker M, van Os J, Wichers M. Mindfulness training increases momentary positive emotions and reward experience in adults vulnerable to depression: a randomized controlled trial. J Consult Clin Psychol. 2011;79(5):618.

69. Henderson VP, Clemow L, Massion AO, Hurley TG, Druker S, Hébert JR. The effects of mindfulness-based stress reduction on psychosocial outcomes and quality of life in early-stage breast cancer patients: a randomized trial. Breast Cancer Res Treat. 2012;131(1):99-109.
70. Garland EL, Farb NA, Goldin P, Fredrickson BL. Mindfulness broadens awareness and builds eudaimonic meaning: A process model of mindful positive emotion regulation. Psychol Inq. 2015;26(4):293-314.

71. Froeliger B, Garland EL, Kozink RV, et al. Meditation-State Functional Connectivity (msFC): strengthening of the dorsal attention network and beyond. Evid Based Complement Alternat Med. 2012; 2012:680407. doi:10.1155/2012/680407.

72. Brewer JA, Worhunsky PD, Gray JR, Tang YY, Weber J, Kober H. Meditation experience is associated with differences in default mode network activity and connectivity. Proc Natl Acad Sci U S A. 2011;108(50):20254-20259. doi:10.1073/pnas.1112029108.

73. Garland EL, Froeliger B, Howard MO. Effects of Mindfulness-Oriented Recovery Enhancement on reward responsiveness and opioid cuereactivity. Psychopharmacology (Berl). 2014;231(16):3229-3238. doi:10.1007/s00213-014-3504-7.

74. Verdejo-García A, Bechara A. A somatic marker theory of addiction. Neuropharmacology. 2009;56 Suppl 1:48-62. doi:10.1016/j. neuropharm.2008.07.035.

75. Farb NA, Segal ZV, Anderson AK. Mindfulness meditation training alters cortical representations of interoceptive attention. Soc Cogn Affect Neurosci. 2013;8(1):15-26.

76. Paulus MP, Stewart JL. Interoception and drug addiction. Neuropharmacology. 2014;76 Pt B:342-350. doi:10.1016/j.neuropharm.2013.07.002.

77. Paulus MP, Stewart JL, Haase L. Treatment approaches for interoceptive dysfunctions in drug addiction. Front Psychiatry. 2013;4:137. Available from: http://www.ncbi.nlm.nih.gov/pmc/articles/ PMC3798869/. Accessed February 17, 2016.

78. Farb NAS, Segal ZV, Anderson AK. Attentional modulation of primary interoceptive and exteroceptive cortices. Cereb Cortex. 2013;23(1):114-126. doi:10.1093/cercor/bhr385.

79. Schooler JW, Smallwood J, Christoff K, Handy TC, Reichle ED, Sayette MA. Meta-awareness, perceptual decoupling and the wandering mind. Trends Cogn Sci. 2011;15(7):319-326. doi:10.1016/j. tics.2011.05.006.

80. Tang YY, Lu Q, Geng X, Stein EA, Yang Y, Posner MI. Short-term meditation induces white matter changes in the anterior cingulate. Proc Natl Acad Sci. 2010;107(35):15649-15652.

81. Tang YY, Lu Q, Fan M, Yang Y, Posner MI. Mechanisms of white matter changes induced by meditation. Proc Natl Acad Sci. 2012;109(26):10570-10574.
Neuroscience and Neuroeconomics

\section{Publish your work in this journal}

Neuroscience and Neuroeconomics is an international, peer-reviewed, open access journal focusing on the identification of brain structures and measurement of neural activity related to behavior, behavioral predictions, and decision making in health and disease. The manuscript

\section{Dovepress}

management system is completely online and includes a very quick and fair peer-review system. Visit http://www.dovepress.com/testimonials. php to read real quotes from published authors. 